\title{
A CENTRAL SCHEME FOR SHALLOW WATER FLOWS ALONG CHANNELS WITH IRREGULAR GEOMETRY
}

\author{
Jorge BALBÁS ${ }^{1}$ AND SMADAR KARNI ${ }^{2}$
}

\begin{abstract}
We present a new semi-discrete central scheme for one-dimensional shallow water flows along channels with non-uniform rectangular cross sections and bottom topography. The scheme preserves the positivity of the water height, and it is preserves steady-states of rest (i.e., it is wellbalanced). Along with a detailed description of the scheme, numerous numerical examples are presented for unsteady and steady flows. Comparison with exact solutions illustrate the accuracy and robustness of the numerical algorithm.
\end{abstract}

Mathematics Subject Classification. 65M99, 35L65.

Received September 25, 2007. Revised June 27, 2008.

Published online December 17, 2008.

\section{The Shallow-WATER MODEL}

We consider the shallow water equations along channels with non-uniform rectangular cross sections and bottom topography. The model describes flows that are nearly horizontal and can be obtained by averaging the Euler equations over the channel cross section [7], resulting in the balance law

$$
\begin{aligned}
& \frac{\partial A}{\partial t}+\frac{\partial Q}{\partial x}=0 \\
& \frac{\partial Q}{\partial t}+\frac{\partial}{\partial x}\left(\frac{Q^{2}}{A}+\frac{1}{2} g \sigma h^{2}\right)=\frac{1}{2} g h^{2} \sigma^{\prime}-g \sigma h B^{\prime},
\end{aligned}
$$

where $h$ and $\sigma(x)$ are, respectively, the height of the fluid above the bottom of the channel, and the channel breadth, $A=\sigma h$ is the wet cross-section, $Q=A u$ is the discharge, with $u$ denoting the (depth average) fluid velocity, $B(x)$ describes the bottom topography of the channel, and $g$ is the acceleration of gravity (see Fig. 1).

Keywords and phrases. Hyperbolic systems of conservation and balance laws, semi-discrete schemes, Saint-Venant system of shallow water equations, non-oscillatory reconstructions, channels with irregular geometry.

${ }^{1}$ Department of Mathematics, California State University, Northridge, CA 91330, USA. jorge.balbas@csun.edu

2 Department of Mathematics, University of Michigan, Ann Arbor, MI 48109, USA. karni@umich.edu 


\subsection{Properties of the system}

The system (1.1) is strictly hyperbolic for $h>0$ and has eigenvalues

$$
\lambda_{1}=u-\sqrt{g h} \quad \text { and } \quad \lambda_{2}=u+\sqrt{g h},
$$

with corresponding eigenvectors

$$
\vec{e}_{1}=\left(\begin{array}{c}
1 \\
u-\sqrt{g h}
\end{array}\right) \quad \text { and } \quad \vec{e}_{2}=\left(\begin{array}{c}
1 \\
u+\sqrt{g h}
\end{array}\right) .
$$

It is endowed with an entropy function

$$
\mathcal{E}(x, t)=\sigma h\left(\frac{1}{2} u^{2}+\frac{1}{2} g h+g B\right)
$$

and satisfies the entropy inequality

$$
\frac{\partial \mathcal{E}}{\partial t}+\frac{\partial}{\partial x}\left[u\left(\mathcal{E}+\frac{1}{2} g \sigma h^{2}\right)\right] \leq 0 .
$$

Smooth steady state solutions to (1.1) satisfy

$$
\begin{aligned}
& Q=\sigma h u=\text { const. } \\
& E=\frac{1}{2} u^{2}+g(h+B)=\text { const. }
\end{aligned}
$$

where, for example, one readily recognizes the trivial steady state of rest

$$
u(x)=0 \quad \text { and } \quad w(x)=h(x)+B(x)=\text { const. }
$$

The parameters $Q$ and $E$ (together with the topography $B(x)$ and channel geometry $\sigma(x)$ ) determine the steadystate solution as the root of a nonlinear equation. A useful relation for smooth steady solutions is obtained by differentiating (1.6)

$$
\left(1-F^{2}\right) h_{x}=\frac{h F^{2}}{\sigma} \sigma^{\prime}-B^{\prime},
$$

where

$$
F=\frac{|u|}{\sqrt{g h}}
$$

is the Froude number. Flows with Froude number $F<1$ are said to be subcritical or fluvial, and flows with Froude number $F>1$ supercritical or torrential. The interplay between the geometry of the channel, $\sigma(x)$, and the topography, $B(x)$, controls the flow. Equation (1.8) implies, for example, that if the crest of the topography $\left(B^{\prime}(x)=0\right)$ and the throat of the channel $\left(\sigma^{\prime}(x)=0\right)$ occur at the same point, then at that point either the solution is symmetric, $h_{x}(x)=0$, or the flow reaches criticality, i.e., $F=1$. If the crest and throat occur at different points in the domain, the flow reaches criticality at some intermediate point where the right hand side of (1.8) vanishes. 

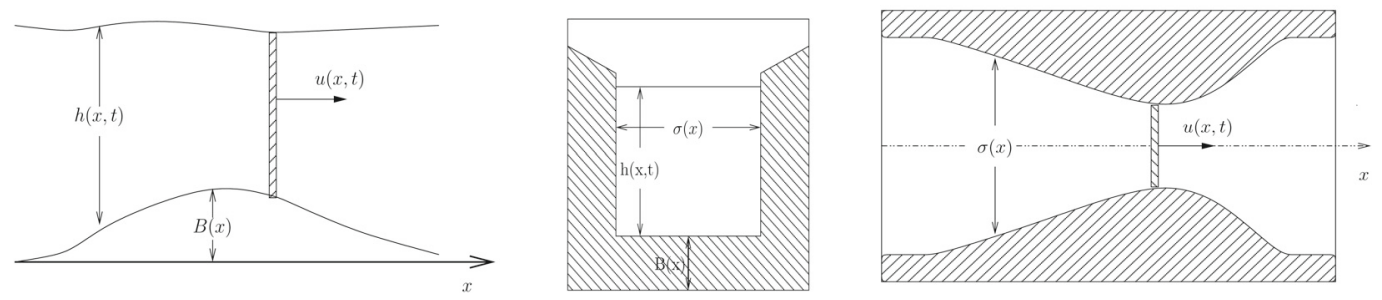

FigurE 1. Three views of the flow. Flow profile (left), flow cross section (center) and overview of flow (right).

\subsection{Numerical simulation of shallow-water flows}

Numerical approximations of the solutions of (1.1) face numerous challenges: the system (1.1) admits discontinuous solutions, and require robust numerical schemes that are suitable for discontinuous flows.

Time changes in the solution of (1.1) arise when flux gradients are out of balance with the geometric source terms. Numerical schemes that are able to recognize and respect such a balance often give superior results when computing near steady-state flows [2]. Perfectly recognizing such a balance may not always be possible, and schemes that respect steady-state solutions either exactly or to the order of the numerical approximation are often called 'well-balanced'. Recent years have seen growing interest in developing well-balanced schemes in various numerical frameworks (see, for example, [6,10,12-14,17,19,21,22]). See also the recent book [5] and references cited therein.

Another challenge arises when computing near dry-state solutions (e.g., flooding or drainage flows) as system (1.1) loses strict hyperbolicity if $h=0$. In such cases, the truncation error of the numerical scheme may cause the layer depth $h$ to become negative, causing the computation to fail. Positivity preserving schemes have the desirable property that if the data has positive (non-negative) depth, so does the numerical solution. Positive schemes enjoy enhanced stability near dry states.

In this paper, we introduce a semi-discrete central scheme for the solution of (1.1). The scheme is second order accurate, well-balanced and positive. Nonoscillatory schemes based on central differencing offer a robust, yet simple, approach for computing the discontinuous solutions of hyperbolic problems, (see, for example, $[3,15,18]$ ). Several central schemes for hyperbolic conservation laws have been extended to handle systems with geometric source terms, in [23] Russo introduces a fully-discrete, well-balanced central scheme for flows along channels of constant width, and in [13,14] several semi-discrete central schemes for one- and two-dimensional shallow water flows are presented. Higher order ENO/WENO based algorithms are given in $[8,28]$ and more recently in $[19,20]$ using the equilibrium variables $Q$ and $E$. Upwind schemes for the treatment of balance laws include [4,6,17,21], and kinetic approaches include [2,22]. The central scheme described in this work extends the work in [13,14,23] to flows in variable geometry. Not many works address shallow water systems in variable geometries. Among those we note the work in [27] using an upwind scheme for single layer shallow water, and [7] where the two-layer shallow water system is solved using the $Q$-scheme [6], the scheme in [8,28], based on a CWENO reconstruction, and the relaxation scheme for two-layer shallow-water presented in [1].

The proposed scheme is described in Section 2, and is established to preserve positivity of water height and to be well-balanced. Numerical solutions are presented in Section 3 for a variety of flow regimes, illustrating the scheme's accuracy and robustness and demonstrating its ability to simulate a wide range of flows.

\section{A Central SCheme For One-Dimensional SHAllow WATER Flows}

In this section we construct a central scheme for the accurate simulation of shallow water flows described by the balance law (1.1). In particular, we seek a scheme that is positivity preserving and well-balanced. The scheme extends previous works in $[13,14,23]$ to shallow water flows in variable geometry. This extension is not 
trivial; in the constant channel width model $(\sigma \equiv 1)$, well-balancing may be accomplished solely by choosing an appropriate discretization of the source term. In the variable geometry case, the conserved variables $\sigma h$ and $\sigma h u$ depend on the geometry $\sigma$, which renders steady state preservation and positivity more strongly coupled with, for example, the reconstruction of the conserved variables.

Following [23], we reformulate (1.1) in terms of the total water elevation $w=h+B$ and the total area, $A^{T}=A+\sigma B=\sigma w$,

$$
\begin{aligned}
& \frac{\partial A^{T}}{\partial t}+\frac{\partial Q}{\partial x}=0 \\
& \frac{\partial Q}{\partial t}+\frac{\partial}{\partial x}\left(\frac{Q^{2}}{A^{T}-\sigma B}+\frac{1}{2} g \sigma(w-B)^{2}\right)=\frac{1}{2} g \sigma^{\prime}(w-B)^{2}-g \sigma(w-B) B^{\prime}
\end{aligned}
$$

This formulation allows the numerical scheme to detect changes (or the lack of them) in the total water elevation $w$, which in turn, facilitates ensuring preservation of steady states of rest.

\subsection{Semi-discrete central formulation}

We begin by describing the semi-discrete central scheme framework for approximating solutions for hyperbolic conservation laws (consult $[15,16]$ for further details),

$$
v_{t}+f(v)_{x}=0 .
$$

For a fixed spatial scale $\Delta x$, we consider the partition of the solution domain into the grid cells $I_{j}:=\left[x_{j}-\right.$ $\left.\Delta x / 2, x_{j}+\Delta x / 2\right]$, and denote by $\bar{v}_{j}(t)$ the cell average of $v(x, t)$ over the cell $I_{j}$,

$$
\bar{v}_{j}(t)=\frac{1}{\Delta x} \int_{x_{j-\frac{1}{2}}}^{x_{j+\frac{1}{2}}} v(x, t) \mathrm{d} x .
$$

Integrating (2.2) over each $I_{j}$ results in the equivalent semi-discrete formulation

$$
\frac{\mathrm{d}}{\mathrm{d} t} \bar{v}_{j}(t)=-\frac{1}{\Delta x}\left(f\left(v\left(x_{j+\frac{1}{2}}, t\right)\right)-f\left(v\left(x_{j-\frac{1}{2}}, t\right)\right)\right) .
$$

Equation (2.3) is approximated by the collection of (semi-discrete) ODEs:

$$
\frac{\mathrm{d}}{\mathrm{d} t} \bar{v}_{j}(t)=-\frac{H_{j+\frac{1}{2}}(t)-H_{j-\frac{1}{2}}(t)}{\Delta x}
$$

where the flux at cell interfaces, $f\left(v\left(x_{j \pm \frac{1}{2}}, t\right)\right)$, is approximated by the numerical flux $H_{j \pm \frac{1}{2}}(t)$ given by

$$
H_{j \pm \frac{1}{2}}(t)=\frac{f\left(v_{j \pm \frac{1}{2}}^{+}(t)\right)+f\left(v_{j \pm \frac{1}{2}}^{-}(t)\right)}{2}-\frac{a_{j \pm \frac{1}{2}}}{2}\left(v_{j \pm \frac{1}{2}}^{+}(t)-v_{j \pm \frac{1}{2}}^{-}(t)\right) .
$$

Here, the interface point-values of the solution, $v_{j \pm \frac{1}{2}}^{ \pm}(t)$, are recovered from the cell averages $\left\{\bar{v}_{j}(t)\right\}$ via a non-oscillatory polynomial reconstruction $v(x, t) \approx R(x ; t)=\sum_{j} p_{j}(x ; t) \cdot \mathbf{1}_{I_{j}}$, i.e.,

$$
v_{j+\frac{1}{2}}^{-}:=p_{j}\left(x_{j+\frac{1}{2}}\right) \quad \text { and } \quad v_{j+\frac{1}{2}}^{+}:=p_{j+1}\left(x_{j+\frac{1}{2}}\right),
$$

and $a_{j+\frac{1}{2}}$ stands for (an estimate of) the maximum wave speed of the conservation law, (2.2), at the cell interface $x_{j+\frac{1}{2}}$, given by the spectral radius of the Jacobian matrix of $f(v), \partial f / \partial v$. 
This semi-discrete formulation, (2.4), and its central-upwind sequel, [16], provide a general framework for non-oscillatory central schemes, requiring for their actual implementation two ingredients: $(i)$ a non-oscillatory polynomial reconstruction of the interface values $\left\{v_{j \pm \frac{1}{2}}^{ \pm}\right\}_{j}$ from their cell averages $\left\{\bar{v}_{j}(t)\right\}_{j}$, and $(i i)$ an evolution routine (i.e., an ODE solver) to update these cell averages according to (2.4).

In analogy, the semi-discrete formulation for the balance law

$$
v_{t}+f(v)_{x}=S(v, x)
$$

yields the semi-discrete system

$$
\frac{\mathrm{d}}{\mathrm{d} t} \bar{v}_{j}(t)=-\frac{H_{j+\frac{1}{2}}(t)-H_{j-\frac{1}{2}}(t)}{\Delta x}+\frac{1}{\Delta x} \int_{x_{j-\frac{1}{2}}}^{x_{j+\frac{1}{2}}} S(v, x) \mathrm{d} x .
$$

For the shallow water system, (2.1),

$$
v=\left(\begin{array}{c}
A^{T} \\
Q
\end{array}\right)=\left(\begin{array}{c}
\sigma w \\
\sigma(w-B) u
\end{array}\right), \quad f(v)=\left(\begin{array}{c}
\sigma(w-B) u \\
\sigma(w-B) u^{2}+\frac{1}{2} g \sigma(w-B)^{2}
\end{array}\right)
$$

and

$$
S(v, x)=\left(\begin{array}{c}
0 \\
\frac{1}{2} g \sigma^{\prime}(w-B)^{2}-g \sigma(w-B) B^{\prime}
\end{array}\right) .
$$

In addition to the non-oscillatory polynomial reconstruction and the evolution routine needed for the implementation of (2.4), the approximation of balance laws requires the discretization of the source term, $S(v, x)$, in (2.8).

\subsection{Non-oscillatory second-order reconstruction}

In order to recover the interface values $v_{j \pm \frac{1}{2}}^{ \pm}(t)$ in $(2.5)$ from the cell averages $\bar{v}_{j}(t)$, we employ a piece-wise linear reconstruction,

$$
v(x, t)=R(x ; \bar{u}(t)):=\sum_{j} p_{j}(x) \cdot \mathbf{1}_{I_{j}}(x) .
$$

This reconstruction procedure is at the heart of high-resolution non-oscillatory central schemes, and requires the coefficients of the polynomials $\left\{p_{j}(x)\right\}$ to be determined so that $R(x ; \bar{v}(t))$ satisfies the following three essential properties:

- $\mathcal{P}_{1}$ - Conservation of cell averages: $\bar{p}_{j}(x)=\bar{v}_{j}(t)$;

- $\mathcal{P}_{2}-$ Accuracy: $R(x ; \bar{v}(t))=v(x, t)+\mathcal{O}\left((\Delta x)^{2}\right)$;

- $\mathcal{P}_{3}$ - Non-oscillatory behavior of $\sum_{j} p_{j}(x)$.

For the shallow water system, (2.1) we also require that the reconstructed values of the total area, $A^{T}$, and the corresponding values of the total water height, $w$, satisfy the following properties:

- $\mathcal{P}_{4}$ - Flux gradient and source balancing: for steady-sates of rest, the interface values of the water height, $h$, must satisfy

$$
h_{j+\frac{1}{2}}^{-}-h_{j-\frac{1}{2}}^{+}=-\left(B\left(x_{j+\frac{1}{2}}\right)-B\left(x_{j-\frac{1}{2}}\right)\right) .
$$

- $\mathcal{P}_{5}$ - Positivity: the reconstructed values $w_{j \pm \frac{1}{2}}^{ \pm}(t)$ must yield $h_{j \pm \frac{1}{2}}^{ \pm}(t) \geq 0$, so as to ensure the positivity of $\bar{h}_{j}(t+\Delta t)$. 


\subsubsection{Minmod reconstruction: properties $\mathcal{P}_{1}-\mathcal{P}_{3}$}

To guarantee properties $\mathcal{P}_{1}-\mathcal{P}_{3}$, we employ a second-order minmod reconstruction [11,26]. The total area, $A^{T}$, and the discharge, $Q$, are reconstructed from their cell averages as the piecewise linear functions (the reconstruction is applied to values at time $t$, thus we can avoid the explicit reference to the time variable for its description)

with the slopes $v_{j}^{\prime}$ calculated as

$$
p_{j}(x)=\bar{v}_{j}+v_{j}^{\prime}\left(x-x_{j}\right),
$$

$$
v_{j}^{\prime}=\frac{1}{\Delta x} \operatorname{minmod}\left(\alpha \Delta_{-} \bar{v}_{j}, \Delta_{0} \bar{v}_{j}, \alpha \Delta_{+} \bar{v}_{j}\right)
$$

where $1 \leq \alpha<2$, and

$$
\operatorname{minmod}\left(x_{1}, x_{2}, x_{3}, \ldots, x_{k}\right)=\left\{\begin{array}{cccc}
\min _{j}\left(x_{j}\right) & \text { if } & x_{j}>0 \quad \forall j \\
\max _{j}\left(x_{j}\right) & \text { if } & x_{j}<0 \quad \forall j \\
0 & & \text { otherwise }
\end{array}\right.
$$

\subsubsection{Reconstruction of height: properties $\mathcal{P}_{4}$ and $\mathcal{P}_{5}$}

In order to enforce property $\mathcal{P}_{4}$ and obtain a well-balanced scheme, we first recover the cell averages of $w$ from those of $A^{T}$ as

$$
\bar{w}_{j}:=\frac{\bar{A}_{j}^{T}}{\sigma_{j}} .
$$

For the flows calculated in Section 3 below, the initial conditions are given for $u$ (or $Q$ ) and $w$, allowing us to define $\bar{A}_{j}^{T}:=\sigma\left(x_{j}\right) \bar{w}_{j}$. Other values can be chosen within the second order accuracy of the scheme (e.g., $\sigma_{j}=\bar{\sigma}_{j}$ ).

The interface values $w_{j \pm \frac{1}{2}}^{\mp}$ are then obtained using the minmod reconstruction (2.13)-(2.15).

To ensure the positivity of $h_{j \pm \frac{1}{2}}^{\mp}$, we follow [14], and limit the slope of the reconstructed values of $w$ as follows (see Fig. 2):

$$
\begin{array}{ll}
\text { if } \quad w_{j-\frac{1}{2}}^{+}<B\left(x_{j-\frac{1}{2}}\right), \quad \text { then set } \quad w_{j}^{\prime}:=2\left(\bar{w}_{j}-B\left(x_{j-\frac{1}{2}}\right)\right), \\
\Rightarrow \quad w_{j-\frac{1}{2}}^{+}=B\left(x_{j-\frac{1}{2}}\right), \quad \text { and } \quad w_{j+\frac{1}{2}}^{-}=\bar{w}_{j}+\frac{1}{2} w_{j}^{\prime},
\end{array}
$$

or

$$
\begin{aligned}
& \text { if } \quad w_{j+\frac{1}{2}}^{-}<B\left(x_{j+\frac{1}{2}}\right), \quad \text { then set } \quad w_{j}^{\prime}:=2\left(B\left(x_{j+\frac{1}{2}}\right)-\bar{w}_{j}\right), \\
& \Rightarrow \quad w_{j+\frac{1}{2}}^{-}=B\left(x_{j+\frac{1}{2}}\right), \quad \text { and } \quad w_{j-\frac{1}{2}}^{+}=\bar{w}_{j}-\frac{1}{2} w_{j}^{\prime} .
\end{aligned}
$$

This linear reconstruction of $w$ clearly satisfies property $\mathcal{P}_{5}, w_{j+\frac{1}{2}}^{ \pm} \geq B\left(x_{j+\frac{1}{2}}\right)$, which in turn will guarantee the positivity of $\bar{h}_{j}(t+\Delta t)$, whose interface values are defined as

$$
\begin{aligned}
h_{j+\frac{1}{2}}^{-} & :=w_{j+\frac{1}{2}}^{-}-B\left(x_{j+\frac{1}{2}}\right) \\
h_{j-\frac{1}{2}}^{+} & :=w_{j-\frac{1}{2}}^{+}-B\left(x_{j-\frac{1}{2}}\right),
\end{aligned}
$$

which satisfy (2.12) when $w_{j-\frac{1}{2}}^{+}=w_{j+\frac{1}{2}}^{-}=w$ as required for well balance. 


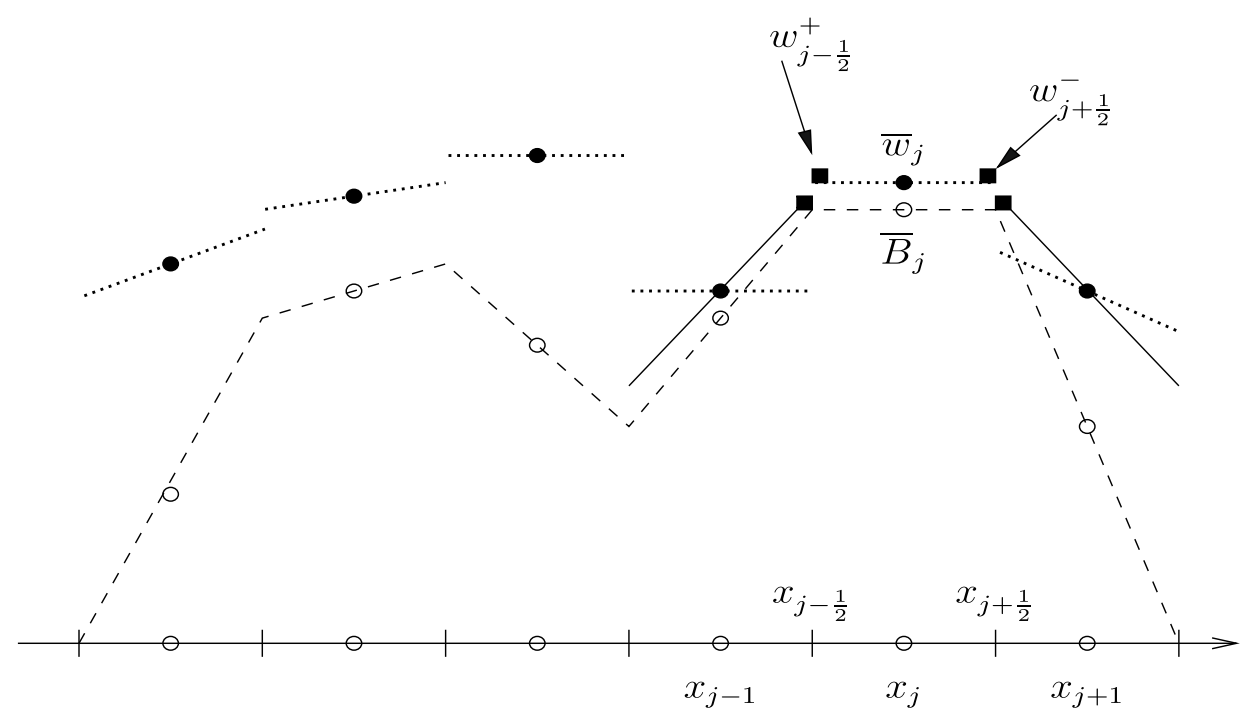

FIGURE 2. Modified reconstruction of total water height, $w$, over the piecewise linear approximation of bottom topography (dashed line). The minmod reconstruction is depicted by dotted lines over cell averages (black dots), the modified reconstruction is depicted by a black solid line, the interface pointvalues of $w, w_{j \pm \frac{1}{2}}$, are depicted by black squares.

\subsection{Balance: discretization of the source term and preservation of steady state}

In the context of shallow water systems, a useful guiding principle in the discretization of the source term in (1.1b) and its cell average in (2.8) is that the resulting scheme is able to recognize and respect steady state solutions. Analytically, such solutions are characterized by a perfect balance between the flux gradient and the source term. Ideally, this property should be inherited by the scheme. In practice, a discrete perfect balance may not be possible to achieve for general steady states, the following discretization respects steady states of rest exactly and more general steady states are respected to the order of the numerical approximation.

For steady states of rest $(u=0, h+B=$ const.), the second component of the numerical flux in (2.8) reduces to

$$
H_{j \pm \frac{1}{2}}(t)=\frac{f\left(v_{j \pm \frac{1}{2}}^{+}\right)+f\left(v_{j \pm \frac{1}{2}}^{-}\right)}{2}=f\left(v_{j \pm \frac{1}{2}}\right)=\frac{1}{2} g \sigma_{j \pm \frac{1}{2}} h_{j \pm \frac{1}{2}}^{2}
$$

and the flux difference term in $(2.8)$ reads

$$
-\frac{H_{j+\frac{1}{2}}(t)-H_{j-\frac{1}{2}}(t)}{\Delta x}=\frac{g}{2 \Delta x}\left(\sigma_{j+\frac{1}{2}} h_{j+\frac{1}{2}}^{2}(t)-\sigma_{j-\frac{1}{2}} h_{j-\frac{1}{2}}^{2}(t)\right) .
$$

We write the right hand side of (2.21) as

$$
\begin{aligned}
& \frac{g}{2 \Delta x}\left(\sigma_{j+\frac{1}{2}} h_{j+\frac{1}{2}}^{2}-\sigma_{j-\frac{1}{2}} h_{j-\frac{1}{2}}^{2}\right)= \\
& \quad \frac{g}{2 \Delta x}\left[\frac{1}{2}\left(\sigma_{j+\frac{1}{2}}-\sigma_{j-\frac{1}{2}}\right)\left(h_{j+\frac{1}{2}}^{2}+h_{j-\frac{1}{2}}^{2}\right)+\frac{1}{2}\left(\sigma_{j+\frac{1}{2}}+\sigma_{j-\frac{1}{2}}\right)\left(h_{j+\frac{1}{2}}^{2}-h_{j-\frac{1}{2}}^{2}\right)\right] .
\end{aligned}
$$


And, in view of this, discretize the cell average of the source term, (2.10), with the approximations

$$
\frac{g}{\Delta x} \int_{x_{j-\frac{1}{2}}}^{x_{j+\frac{1}{2}}} \frac{1}{2} \sigma^{\prime} h^{2} \mathrm{~d} x \approx \frac{g}{2} \frac{\sigma_{j+\frac{1}{2}}-\sigma_{j-\frac{1}{2}}}{\Delta x} \frac{\left(h_{j+\frac{1}{2}}^{-}\right)^{2}+\left(h_{j-\frac{1}{2}}^{+}\right)^{2}}{2}
$$

and

$$
-\frac{g}{\Delta x} \int_{x_{j-\frac{1}{2}}}^{x_{j+\frac{1}{2}}} \sigma h B^{\prime} \mathrm{d} x \approx-g \frac{\sigma_{j+\frac{1}{2}}+\sigma_{j-\frac{1}{2}}}{2} \frac{h_{j+\frac{1}{2}}^{-}+h_{j-\frac{1}{2}}^{+}}{2} \frac{B\left(x_{j+\frac{1}{2}}\right)-B\left(x_{j-\frac{1}{2}}\right)}{\Delta x} .
$$

We observe that the approximation (2.23) exactly balances the first product on the right hand side of $(2.22)$ and, with the interface pointvalues of the water height reconstructed as (2.19), (2.24) exactly balances the second product on the right hand side of (2.22). With this discretization, the right hand side (RHS) of the momentum balance in (2.8) vanishes for steady state of rest.

To ensure preservation of total water height $w$ remains constant over time, i.e., the first component of the numerical fluxes, $H_{j \pm \frac{1}{2}}$, must be calculated so that their difference yields

$$
\frac{\mathrm{d} \bar{A}_{j}^{T}}{\mathrm{~d} t}=0
$$

when $w=$ const. and $u \equiv 0$.

We approximate the interface jump of the total area in the numerical flux of the total area in (2.8) by

$$
A_{j+\frac{1}{2}}^{T,+}-A_{j+\frac{1}{2}}^{T,-} \equiv \sigma\left(x_{j+\frac{1}{2}}\right)\left(w\left(x_{j+\frac{1}{2}}^{+}, t\right)-w\left(x_{j+\frac{1}{2}}^{-}, t\right)\right) \approx \sigma_{j+\frac{1}{2}}\left(w_{j+\frac{1}{2}}^{+}-w_{j+\frac{1}{2}}^{-}\right)
$$

where

with

$$
\sigma_{j+\frac{1}{2}}=\max \left\{\sigma_{j+\frac{1}{2}}^{-}, \sigma_{j+\frac{1}{2}}^{+}\right\}
$$

\section{Remarks.}

$$
\sigma_{j+\frac{1}{2}}^{-}:=\frac{A_{j+\frac{1}{2}}^{T,-}}{w_{j+\frac{1}{2}}^{-}} \quad \text { and } \quad \sigma_{j+\frac{1}{2}}^{+}:=\frac{A_{j+\frac{1}{2}}^{T,+}}{w_{j+\frac{1}{2}}^{+}}
$$

1. Approximating $A_{j+\frac{1}{2}}^{T,+}-A_{j+\frac{1}{2}}^{T,-}$ by (2.26) ensures that this term vanishes for steady states of rest as required.

2. The choice of $\sigma_{j \pm \frac{1}{2}}$ in (2.26) ensures positive values of the cell average of the water height $\bar{h}_{j}(t+\Delta t)$ (see Appendix); other choices are possible within the second order accuracy of the scheme.

\subsection{Time evolution}

Given the reconstructed interface values at time $t$ as described in Section 2.2,

$$
v_{j \pm \frac{1}{2}}^{ \pm}(t)=\left(\begin{array}{c}
A_{j \pm \frac{1}{2}}^{ \pm}(t) \\
Q_{j \pm \frac{1}{2}}^{ \pm}(t)
\end{array}\right)
$$

we estimate the maximum interface wave speeds of (2.1) as

$$
a_{j \pm \frac{1}{2}}=\max \left\{\left|u_{j \pm \frac{1}{2}}^{-}\right|+\sqrt{g h_{j \pm \frac{1}{2}}^{-}},\left|u_{j \pm \frac{1}{2}}^{+}\right|+\sqrt{g h_{j \pm \frac{1}{2}}^{+}}\right\}
$$


and the ODE system (2.8) is numerically integrated using the second order Strong Stability Preserving RungeKutta scheme, $[9,24]$,

$$
\begin{aligned}
v^{(1)} & =v^{(0)}+\Delta t C\left[v^{(0)}\right], \\
v^{(2)} & =v^{(1)}+\frac{\Delta t}{2}\left(C\left[v^{(1)}\right]+C\left[v^{(0)}\right]\right), \\
\bar{v}(t+\Delta t) & :=v^{(2)}
\end{aligned}
$$

with the numerical fluxes

$$
C[v(t)]=-\frac{H_{j+\frac{1}{2}}(v(t))-H_{j-\frac{1}{2}}(v(t))}{\Delta x}+\bar{S}_{j}(t)
$$

where $H_{j \pm \frac{1}{2}}$ is given by (2.5), using (2.26), and the cell average of the source term, $\bar{S}_{j}$, approximated by $(2.23)-(2.24)$.

\subsection{Properties of the scheme and additional remarks}

To conclude this section, we summarize the two main properties of the semi-discrete central scheme resulting from applying the discretization of the source term (2.23)-(2.24), along with the reconstruction (2.13)-(2.19), and the SSP Runge-Kutta solver (2.31)-(2.32) to the semi-discrete central formulation (2.8). (We defer the proofs of the following theorems to Appendix.)

Theorem 2.1. Consider the balance law (2.1) and the semi-discrete central formulation (2.8) with the spatial integral of the source term approximated by (2.23)-(2.24), the interface pointvalues of $w(x, t)$ and $h(x, t)$, given respectively by (2.17)-(2.18) and (2.19), those of $A^{T}(x, t), Q(x, t)$ recovered by the minmod reconstruction (2.13)-(2.15), and the jump of the total area across the cell interfaces approximated by (2.26)-(2.27). Then

(i) the system of ODEs (2.8) satisfies

$$
\frac{\mathrm{d}}{\mathrm{d} t}\left(\begin{array}{c}
\bar{A}_{j}^{T}(t) \\
\bar{Q}_{j}(t)
\end{array}\right)=0 \quad \forall j,
$$

for $w=$ const. and $u \equiv 0$, i.e., the central scheme is well-balanced, and

(ii) if the cell averages $\bar{A}^{T}(t)$ are such that

$$
\bar{w}_{j}(t)-\frac{1}{2}\left(B\left(x_{j-\frac{1}{2}}\right)+B\left(x_{j+\frac{1}{2}}\right)\right) \geq 0, \quad \forall j,
$$

then, the cell averages $\bar{A}^{T}(t+\Delta t)$ as evolved with (2.31)-(2.32) under the CFL limitation,

$$
\frac{\Delta t}{\Delta x}<\frac{\sigma_{j}}{2 a_{j}} \quad \forall j
$$

where $a_{j}=\max \left\{a_{j-\frac{1}{2}} \sigma_{j-\frac{1}{2}}, a_{j+\frac{1}{2}} \sigma_{j+\frac{1}{2}}\right\}$, will yield

$$
\bar{w}_{j}(t+\Delta t)-\frac{1}{2}\left(B\left(x_{j-\frac{1}{2}}\right)+B\left(x_{j+\frac{1}{2}}\right)\right) \geq 0, \quad \forall j .
$$


The fluxes $f$ in (2.9) require the pointwise values of the flow velocity $u_{j \pm \frac{1}{2}}^{\mp}$. Recovering the flow velocity via $Q / A$ may be inaccurate when $h$ (hence both $Q$ and $A$ ) is very small, and may lead to instabilities. To prevent this, we follow the de-singularization strategy proposed in [14], and compute the flow velocity, $u$, according to

$$
u=\frac{\sqrt{2} A Q}{\sqrt{A^{4}+\max \left(A^{4}, \epsilon\right)}},
$$

with $\epsilon=(\Delta x)^{4}$, and recalculate the discharge $Q:=A \cdot u$ accordingly. When $A$ is small, we must recalculate the discharge as $Q:=A \cdot u$ so as to ensure the well balance and positivity properties (consult [14] for a detailed discussion of this and other desingularization techniques).

\section{NumericAl RESUlts}

In this section we present the numerical solutions for a range of problems illustrating the properties of our central scheme in a variety of flow situations: we validate the ability of the scheme to respect steady states of rest (1.7), and study its ability to propagate small perturbations from rest. The positivity of the scheme is demonstrated by solving several so-called dam-break problems, where a reservoir initially at rest drains through its boundaries. Finally, we study the convergence of transient solutions to more general steady state solutions by comparing them to the exact solutions. As pointed out in Section 2.5, exact steady state solutions are available. These are determined by the parameters $Q$ and $E$, and are obtained by iterative root finding of (1.6) (e.g., using Newton's method).

The flows are calculated along channels with varying width, we have used channels with a parabolic contraction. The contraction is described by the quadratic interpolant through the points $\left(x_{l}, 1\right),\left(\frac{1}{2}\left(x_{l}+x_{r}\right), \sigma_{\min }\right)$, and $\left(x_{r}, 1\right)$, where $x_{l}$, and $x_{r}$ are, respectively, the left and right end-points of the contraction along the $x$-axis, and $\sigma_{\min }$ stands for the minimum width of the channel (with $\sigma_{\min }=1$ corresponding to a straight channel).

For all the results presented below the value of the acceleration of gravity is taken as $g=9.81$, and the time step, $\Delta t$, satisfies the $C F L$ restriction

$$
\Delta t \leq \frac{c \Delta x}{\max _{j} a_{j+\frac{1}{2}}}, \quad c<1
$$

Unless otherwise mentioned, the computations below were performed using 200 grid cells and $c=0.75$.

\subsection{Steady-state of rest and small perturbation from rest}

\subsubsection{The steady-state of rest}

For this problem, the initial conditions correspond to the equilibrium solution,

$$
w(x, 0)=1 \quad \text { and } \quad u(x, 0)=0,
$$

with the bottom topography given by

$$
B(x)=\left\{\begin{array}{cc}
\frac{1}{4}\left(1+\cos \frac{\pi(x-0.5)}{0.1}\right) & \text { if } \quad x \in[0.4,0.6] \\
0 & \text { otherwise. }
\end{array}\right.
$$

Computed solutions are shown in Figure 3 for different channel geometries. Three cases are shown. In all cases, the topography crest is centered at $x=0.5$. In the middle example, the contraction is also centered at $x=0.5$, in the case on the left, the throat slightly precedes the crest, while on the right, the throat is slightly after the crest (see top figures respectively). The steady state of rest is clearly respected by the scheme. 

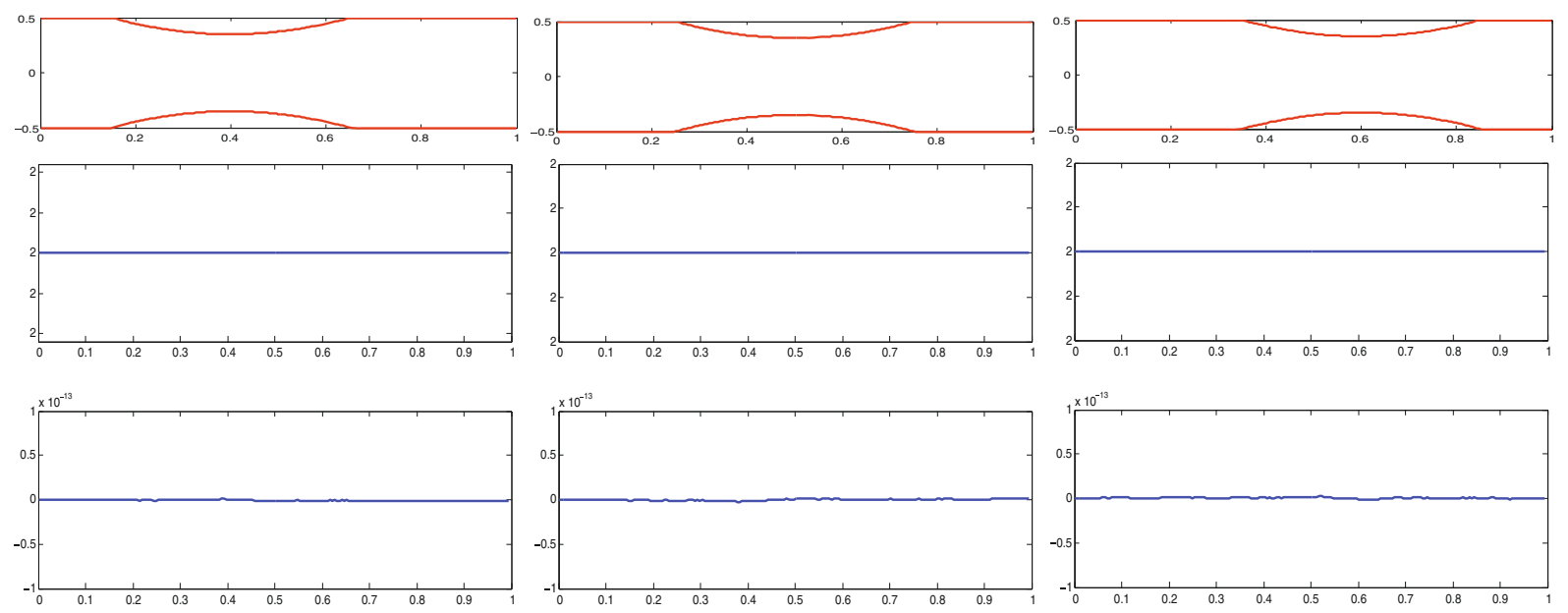

Figure 3. Computation of steady-state of rest through contracting channels. Top: channel longitudinal shape $\left(\sigma_{\min }=0.7\right.$ in all cases $)$, middle: total water height, $w$, bottom: flow velocity, $u$.

\subsubsection{Small perturbations from rest}

For the following problems, the initial conditions correspond to a small perturbation of the steady state of rest,

$$
u(x, 0)=0 \quad \text { and } \quad w(x, 0)= \begin{cases}1+\epsilon & \text { if } 0.1 \leq x \leq 0.2 \\ 1 & \text { otherwise }\end{cases}
$$

Figure 4 shows the initial conditions and several snapshots of the perturbation as it propagates for the case $\epsilon=10^{-2}$. Figure 5 shows the solution at $t=0.25$ with $\epsilon=10^{-5}$ for a channel with a centered contraction. Numerical methods that do not respect steady state of rest have hard time computing accurately the propagation of perturbations of this small magnitude [17]. As can be observed, the perturbation is propagated accurately by the numerical scheme.

\subsection{Drainage following a dam break}

In this problem, we consider a (symmetric) reservoir initially at rest, draining onto a dry bed through its boundaries, leaving behind water trapped in topographical troughs. Due to the symmetry, the flow is computed on half the domain, with open boundary conditions on the right boundary, and reflecting bc's on the left boundary. This flow is computationally challenging as water depth $h$ becomes increasingly small due to drainage, and may lead to breakdown of the solution if water depth becomes negative due to numerical error. The positivity property of the scheme insures the water depth remains non-negative. Two bottom topographies are considered, consisting of one or two topographical bumps. The initial conditions are, in both cases,

$$
u(x, 0)=0, \quad \text { and } \quad w(x, 0)=0.8
$$

At outflow, the boundary conditions are implemented as follows: if the flow is supercritical, both $w$ and $Q$ are extrapolated from the interior of the domain, while if the flow is subcritical, the water height $h_{\text {out }}$ is prescribed to dry bed, $h=0$ - in fact we have used $h_{\text {out }}=10^{-16}$ - and $Q$ is extrapolated. Solution snapshots are presented in Figures 6 and 7. 

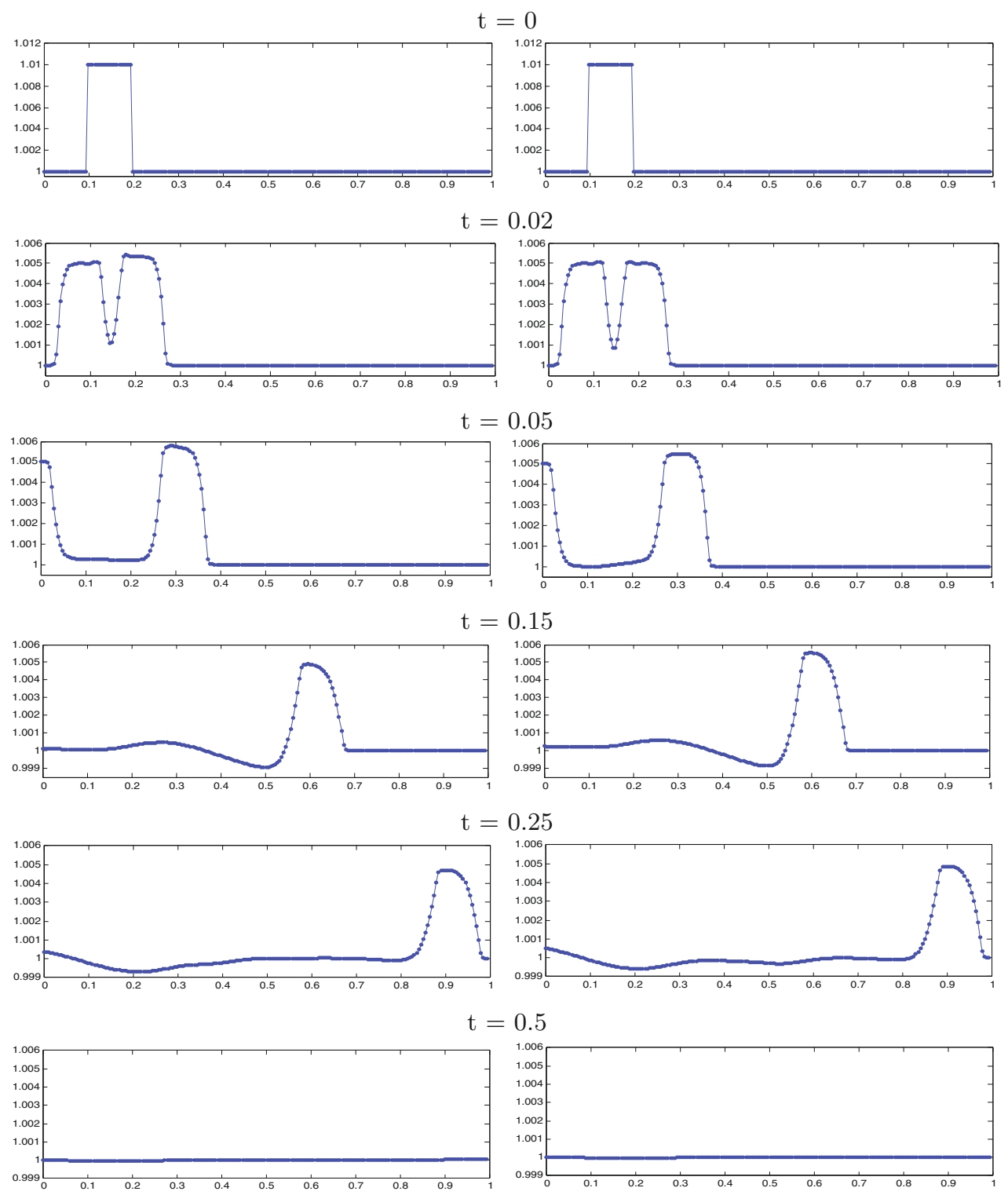

Figure 4. Propagation of small disturbances from rest (3.4) through contracting channels, $\epsilon=10^{-2}$. Left: a channel with a left shifted contraction, right: a channel with a centered contraction.

\subsection{Lake with moving shore}

The next problem, proposed in [2], simulates an oscillating lake with a non-flat bottom and non-vertical shores, providing a valuable numerical test for inundating storm tides [25]. The lake bed extends over the interval $[0,1]$ and its bottom and non-vertical shores are defined by

$$
B(x)=\frac{1}{2}\left[1-\frac{1}{2}(1+\cos 2 \pi(x-0.5))\right],
$$




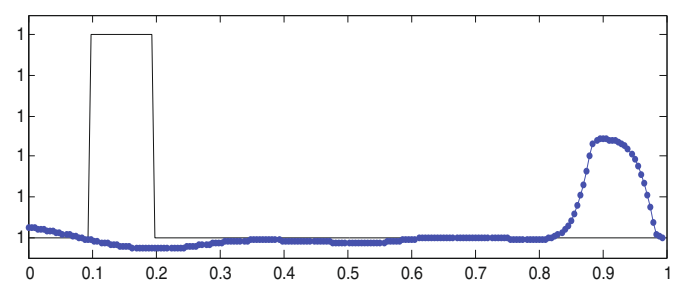

Figure 5. Propagation of small disturbances from rest, (3.4) through contracting channels, $\epsilon=10^{-5}$. Total water height, $w$, at $t=0.25$ (dots) over initial conditions (solid line).

$\mathrm{t}=0$

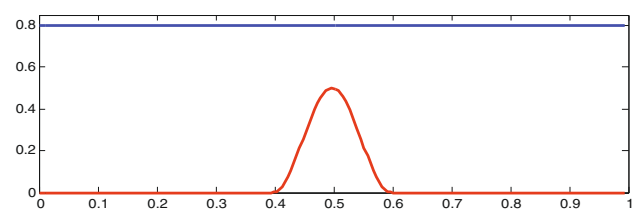

$\mathrm{t}=0.25$

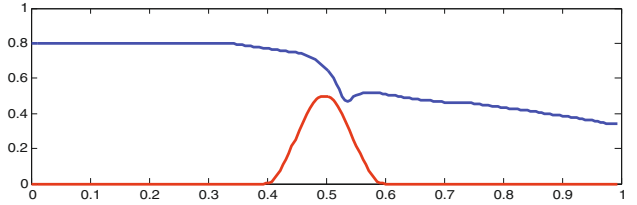

$\mathrm{t}=1$

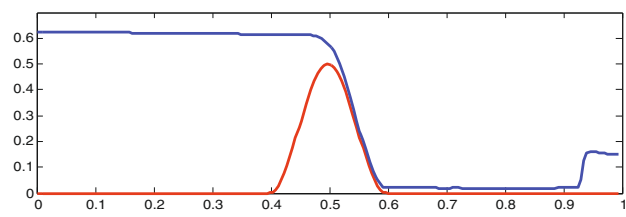

$\mathrm{t}=0.15$

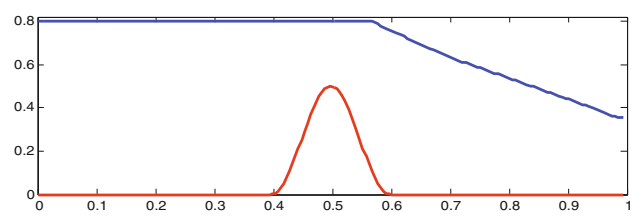

$\mathrm{t}=0.5$

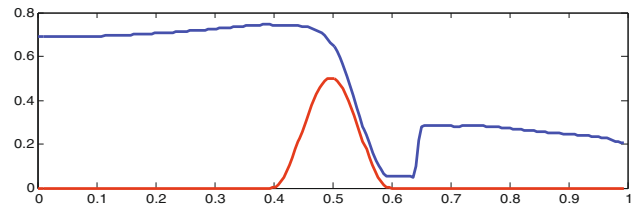

$\mathrm{t}=100$

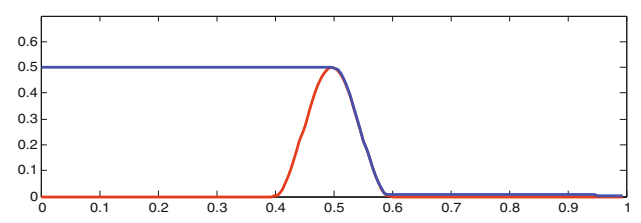

FiguRE 6. Dam break and drainage through a contracting channel: initial conditions (3.5), crest and throat coincide $\sigma^{\prime}(0.5)=B^{\prime}(0.5)=0$. Total water elevation, $w$, at various times.

with a small - centered - parabolic contraction $\left(\sigma_{\min }=0.8\right)$. Initially, the lake is perturbed from rest by a sinusoidal wave that affects the free surface,

$$
w(x, 0)=\max \left\{B(x)+10^{-16}, 0.4+0.04 \sin \kappa(x-0.5)\right\}, \quad \text { with } \kappa=4.0547 .
$$

The flow oscillates over time, creating an interface between a wet cell and a dry cell on each shore of the lake that change their location at each time step. A solution at $t=18.002$ - corresponding to a time where the flow reaches its higher level on the left shore - is presented in Figure 8. The longtime solution computed with the proposed central scheme keeps the periodic regime and preserves the positivity of the water layer.

\subsection{Non-trivial steady-states}

In this section we compute general steady states by integrating the time dependent equations (1.6) for very long time until steady state is reached. The computed solutions are compared with exact steady state solutions. 

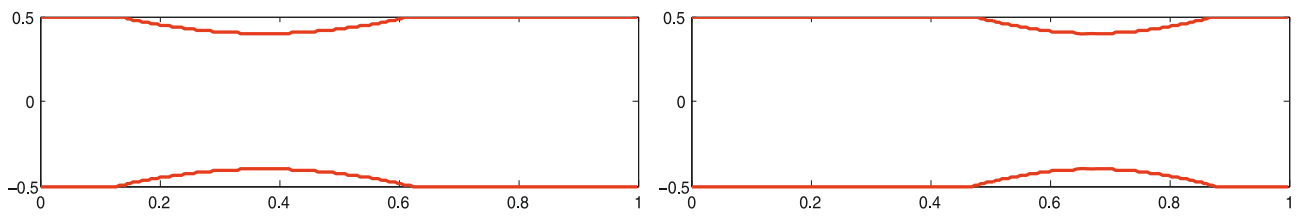

$\mathrm{t}=0.25$
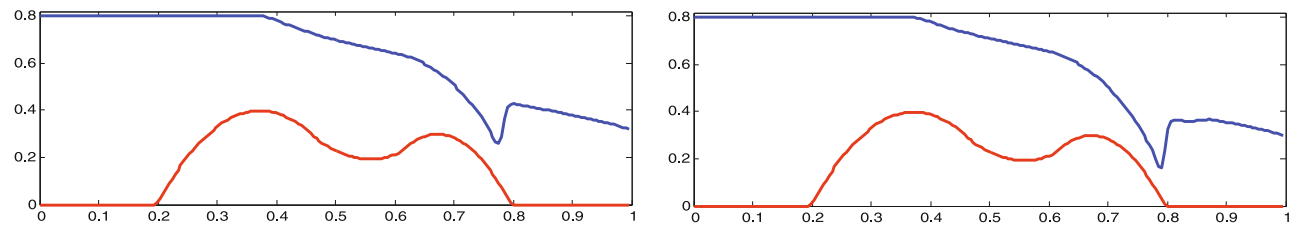

$\mathrm{t}=0.5$
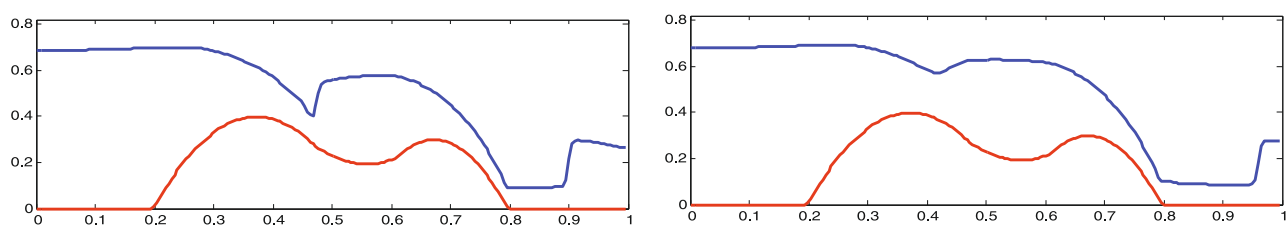

$\mathrm{t}=1$
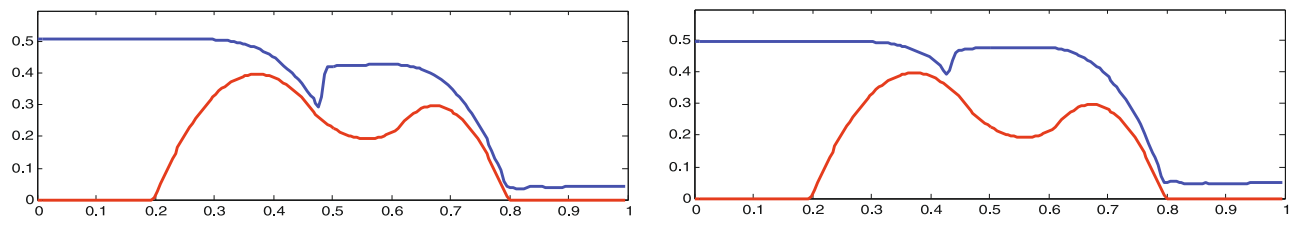

$\mathrm{t}=10$
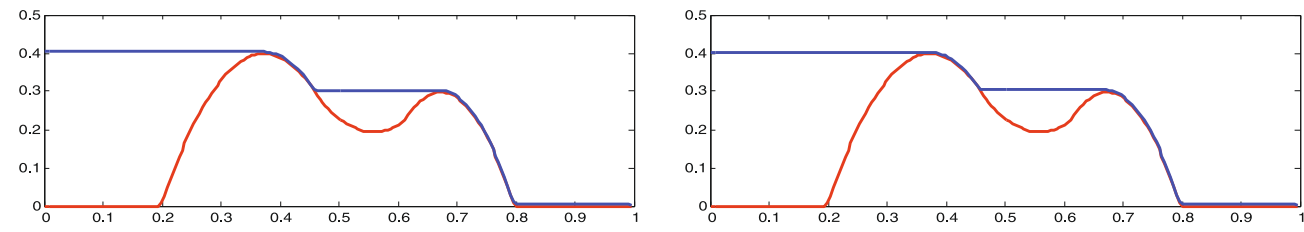

FIGURE 7. Dam break and drainage through a contracting channel with initial conditions (3.5). Left: throat coincides with left crest $\sigma^{\prime}(0.325)=B^{\prime}(0.325)=0$. Right: throat coincides with right crest, $\sigma^{\prime}(0.675)=B^{\prime}(0.675)=0$. Total water elevation, $w$, is shown at various times.

We study various channel configurations, and compare flows in a straight channel to flows in contracting channels. We present computed solutions for a subcritical flow, for a smooth transcritical flow, and for a non-smooth transcritical flow.

For the flows considered here, we consider the solution domain $x \in[-10,10]$, along channels with parabolic contractions as those described above, and with the bottom topography given by

$$
B(x)=\max \left\{0.2-0.05 x^{2}, 0\right\}
$$

The boundary conditions for these steady flows are calculated according to the eigen values of the system, (1.2): at the (left) inflow boundary, the values of $Q$ and $A^{T}$ are extrapolated from the interior of the domain if 


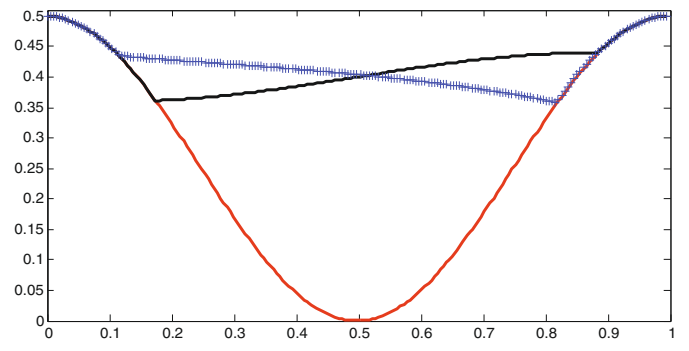

FIGURE 8. Evolution of sinusoidal perturbation of free surface of a lake with non-vertical shores and bottom topography. Total water elevation, $w$ : initial conditions (3.7) (solid line), and at $t=18.002(+)$. The deepest point in topography coincides with center of parabolic contraction, $\sigma^{\prime}(0.5)=B^{\prime}(0.5)=0$.

both eigen values are negative, the value of $Q$ is prescribed and that of $A^{T}$ extrapolated if $\lambda_{1}<0$ and $\lambda_{2}>0$, and both quantities are prescribed if both eigen values are positive. Correspondingly, at the outflow (right) boundary both values are extrapolated when the eigen values are both positive, prescribed when both eigen values are negative, and one is prescribed ( $h_{\text {out }}$ in this case) and one extrapolated $(\mathrm{Q})$ if they have opposite signs.

\subsubsection{Subcritical smooth flow}

For this flow, we take as initial conditions

$$
w(x, 0)=2, \quad \text { and } \quad Q(x, 0)=4.42,
$$

and compute the solution along a channel with a centered contraction $\left(i . e ., \sigma^{\prime}(0)=B^{\prime}(0)=0\right)$. The flow will remain subcritical provided the channel contraction is not too severe $\left(\sigma_{\min }>0.8842\right)$.

The computed large time solutions together with the exact solutions are displayed in Figure 9. To get a sense of the effect of channel contraction on the flow, the solution is also computed in a straight channel. Also shown are the computed solutions for the parameters $Q$ and $E$, the exact values of which are constant in the steady limit. We note that the computed values of $Q$ and $E$ are constant within less than $0.068 \%$ and $0.052 \%$ respectively in the computed solution.

\subsubsection{Transcritical smooth flow}

The steady state solutions in the following examples correspond to flows accelerating smoothly from subcritical $(F<1)$ to supercritical $(F>1)$ through channels with a $30 \%$ contraction. The flow reaches criticality as it runs over the bump and through the contraction at a point between the throat of the channel and the crest of the topography.

For these flows, we take as initial conditions

$$
w(x, 0)=\left\{\begin{array}{ll}
1.31 & \text { if } x<0 \\
0.34 & \text { if } x>0
\end{array} \text { and } \quad Q(x, 0)=1.53 .\right.
$$

Figure 10 displays the computed and exact steady state solutions. For this problem, we have used a $C F L$ number $c=0.6$. Computed values of $Q$ and $E$ are constant within $1.8 \%$.

\subsubsection{Transcritical flow with a shock}

In this example the initial conditions are again (3.9) but the channel has narrower contraction than those considered in the subcritical case $\left(\sigma_{\min }<0.8842\right)$. The steady state solution corresponds to a flow accelerating 

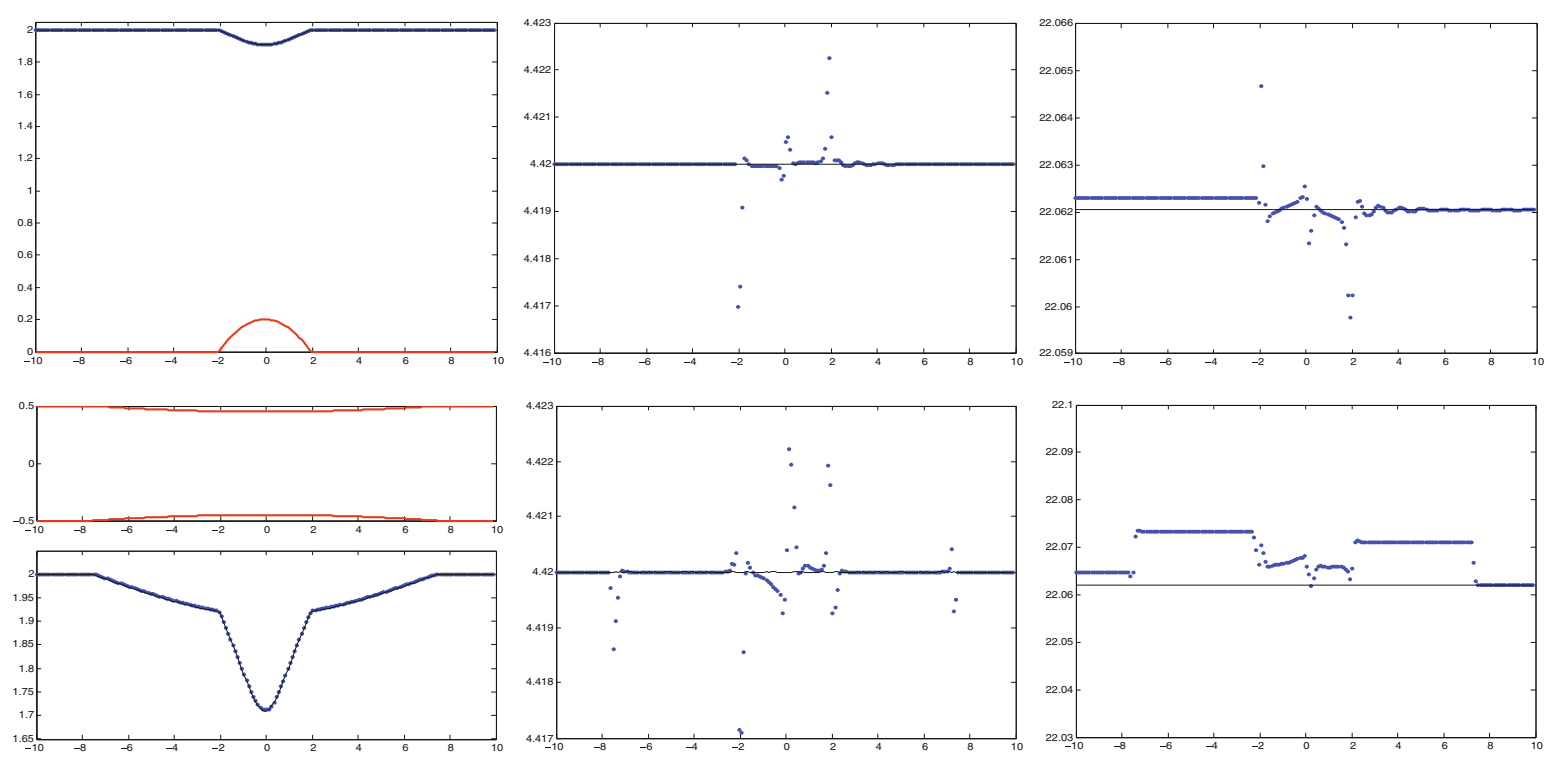

Figure 9. Smooth (subcritical) steady-state flow. Top: straight channel $(\sigma \equiv 1)$, bottom: varying channel $\left(\sigma_{\min }=0.9\right)$. Computed solution (dots) and exact equilibrium solution (solid line). Left: total water elevation, $w=h+B$ and channel geometry, center: discharge, $Q=\sigma h u$, right: total energy, $E$.

from sub- to supercritical as it runs over the bump and through the contraction, then decelerates abruptly to subcritical flow through a shock (hydraulic jump) in order to match the outflow boundary conditions. Solutions corresponding to a centered contraction and a left-shifted contraction are presented in Figure 11. The computed values of $Q$ and $E$ are, respectively, within $4.0 \%$ and $3.7 \%$ of the corresponding (piecewise) constant values.

\section{Appendix: Proof of Theorem 2.1}

\section{Proof.}

(i) Starting with the initial conditions $u(x)=0$ and $w(x)=W$ for all $x$, we fix a space scale $\Delta x$ and the corresponding partition of the solution domain, $\left\{I_{j}\right\}:=\left\{\left[x_{j-\frac{1}{2}}, x_{j+\frac{1}{2}}\right]\right\}$. We then define the cell averages of the conserved quantities in the modified shallow water model, (2.1), as

$$
\bar{A}_{j}^{T}:=\sigma_{j} \bar{w}_{j}=\sigma_{j} W,
$$

and

$$
\bar{Q}_{j}:=\left(\bar{A}_{j}-\frac{1}{2} \sigma_{j}\left(B\left(x_{j+\frac{1}{2}}\right)+B\left(x_{j-\frac{1}{2}}\right)\right)\right) \bar{u}_{j} \equiv 0 .
$$

The reconstructed point values of $w$ and $Q$, clearly satisfy $w_{j \pm \frac{1}{2}}^{ \pm}=W$ and $Q_{j \pm \frac{1}{2}}^{ \pm}=0$, thus the values of the water height, (2.19), and those of the bottom topography at the cell interfaces, $B\left(x_{j \pm \frac{1}{2}}\right)$, satisfy

$$
h_{j+\frac{1}{2}}^{-}-h_{j-\frac{1}{2}}^{+}=-\left(B\left(x_{j+\frac{1}{2}}\right)-B\left(x_{j-\frac{1}{2}}\right)\right) .
$$



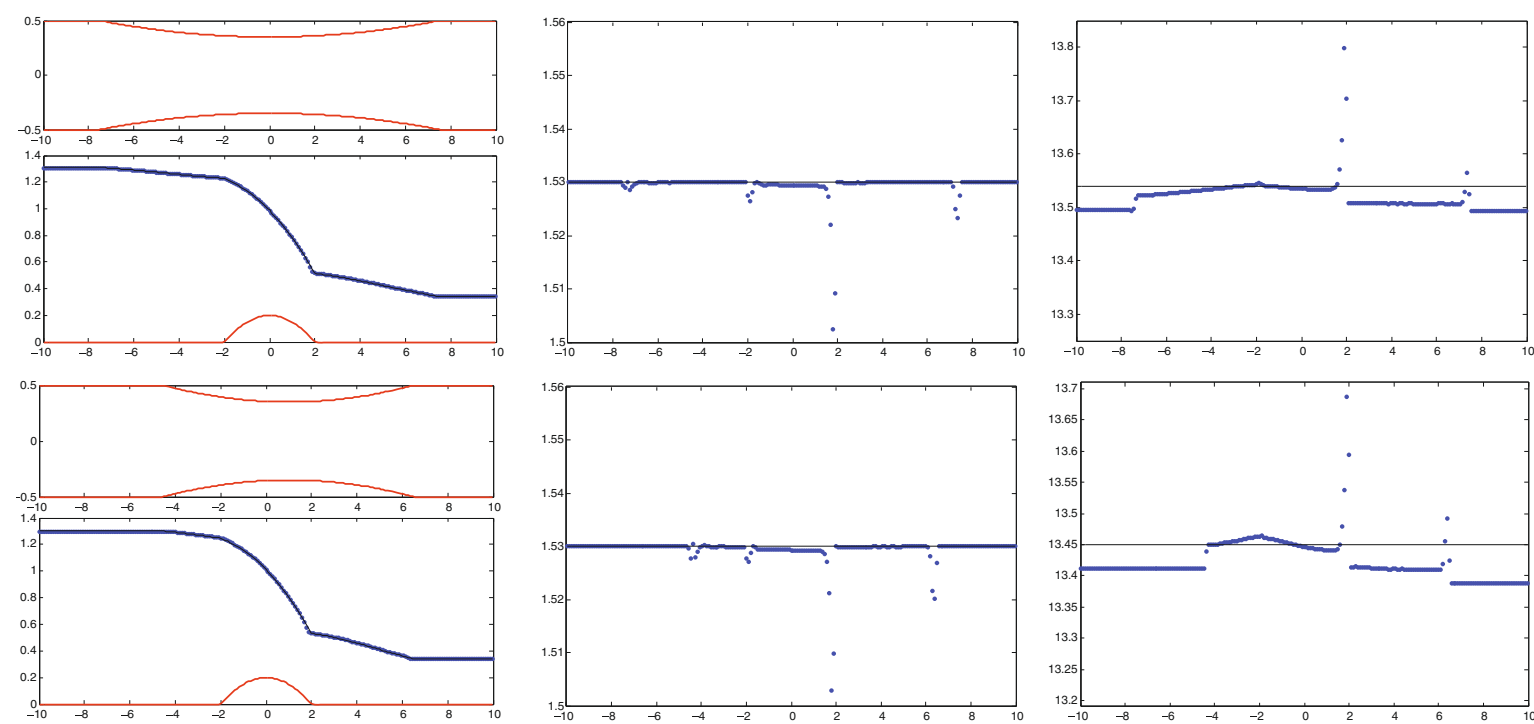

Figure 10. Smooth transcritical steady-state flow in a contracting channel $\left(\sigma_{\min }=0.7\right)$, computed solution (dots) and exact solution (solid line). Top: channel with centered contraction, bottom: channel with right-shifted contraction. Left: total water elevation, $w=h+B$, and channel geometry, center: discharge $Q=\sigma h u$, right: flow energy $E$.
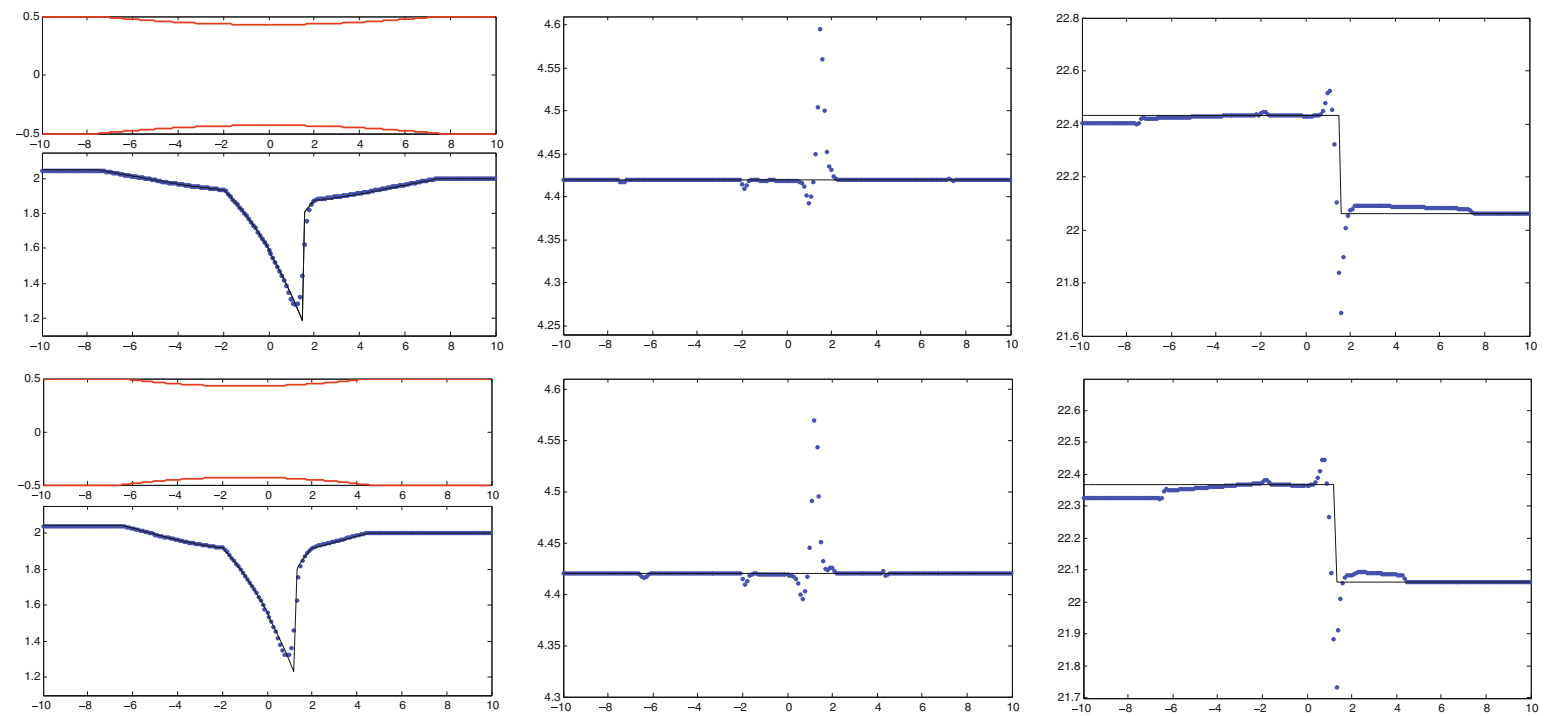

FiguRE 11. Transcritical steady-state flow in a contracting channel $\left(\sigma_{\text {min }}=0.86\right)$, computed solution (dots) and exact solution (solid line). Top: channel with centered contraction, bottom: channel with left-shifted contraction. Left: total water elevation, $w=h+B$, and contraction, center: discharge $Q=\sigma h u$, right: flow energy $E$.

In view of this, the first component of the numerical fluxes $H_{j+\frac{1}{2}}$ in (2.8) reads

$$
-\frac{H_{j+\frac{1}{2}}^{(1)}-H_{j-\frac{1}{2}}^{(1)}}{\Delta x}=-\frac{1}{2 \Delta x}\left[a_{j+\frac{1}{2}} \sigma_{j+\frac{1}{2}}\left(w_{j+\frac{1}{2}}^{+}-w_{j+\frac{1}{2}}^{-}\right)-a_{j-\frac{1}{2}} \sigma_{j-\frac{1}{2}}\left(w_{j-\frac{1}{2}}^{+}-w_{j-\frac{1}{2}}^{-}\right)\right] \equiv 0 .
$$


That is,

$$
\frac{\mathrm{d}}{\mathrm{d} t} \bar{A}_{j}^{T}(t)=0 \quad \Rightarrow \quad \bar{A}_{j}^{T}(t+\Delta t)=\bar{A}_{j}^{T}(t)
$$

which allows us to recover $\bar{w}_{j}(t+\Delta t)=W$ exactly from (3.11).

Noting that, according to (2.19), $h_{j+\frac{1}{2}}^{+}=h_{j+\frac{1}{2}}^{-}=: h_{j+\frac{1}{2}}$, the second component of the numerical flux amounts to (2.20), and since (2.12) holds, it is balanced by (2.23)-(2.24), therefore

$$
\frac{\mathrm{d}}{\mathrm{d} t} \bar{Q}_{j}(t)=0 \quad \Rightarrow \quad \bar{Q}_{j}(t+\Delta t)=\bar{Q}_{j}(t)=0
$$

and $u_{j}(t+\Delta t) \equiv 0$ is also recovered exactly.

(ii) We begin by writing explicitly the cell average $A_{j}^{T}(t+\Delta t)$ when the system $(2.8)$ is evolved with forward Euler's ODE solver,

$$
\bar{A}_{j}^{T}(t+\Delta t)=\bar{A}_{j}^{T}(t)-\lambda\left[H_{j+\frac{1}{2}}^{(1)}(t)-H_{j-\frac{1}{2}}^{(1)}(t)\right],
$$

where $\lambda=\Delta t / \Delta x$. This amounts to

$$
\begin{aligned}
\bar{A}_{j}^{T}(t+\Delta t)= & \bar{A}_{j}^{T}(t)-\frac{\lambda}{2}\left[\left(Q_{j+\frac{1}{2}}^{+}+Q_{j+\frac{1}{2}}^{-}\right)-a_{j+\frac{1}{2}}\left(A_{j+\frac{1}{2}}^{T,+}-A_{j+\frac{1}{2}}^{T,-}\right)\right. \\
& \left.-\left(Q_{j-\frac{1}{2}}^{+}+Q_{j-\frac{1}{2}}^{-}\right)+a_{j-\frac{1}{2}}\left(A_{j-\frac{1}{2}}^{T,+}-A_{j+\frac{1}{2}}^{T,-}\right)\right]
\end{aligned}
$$

(where all the terms on the right hand side are understood to be evaluated at time $t$ ). Using $Q_{j \pm \frac{1}{2}}^{ \pm}=$ $\sigma_{j \pm \frac{1}{2}}^{ \pm} h_{j \pm \frac{1}{2}}^{ \pm}$, we write

$$
\begin{aligned}
\bar{A}_{j}^{T}(t+\Delta t)= & \bar{A}^{T}(t)+\frac{\lambda}{2}\left[\left(a_{j+\frac{1}{2}} \sigma_{j+\frac{1}{2}}-u_{j+\frac{1}{2}}^{+} \sigma_{j+\frac{1}{2}}^{+}\right) h_{j+\frac{1}{2}}^{+}+\left(a_{j-\frac{1}{2}} \sigma_{j-\frac{1}{2}}+u_{j-\frac{1}{2}}^{-} \sigma_{j-\frac{1}{2}}^{-}\right) h_{j-\frac{1}{2}}^{-}\right. \\
& \left.+a_{j+\frac{1}{2}} \sigma_{j+\frac{1}{2}} B\left(x_{j+\frac{1}{2}}\right)+a_{j-\frac{1}{2}} \sigma_{j-\frac{1}{2}} B\left(x_{j-\frac{1}{2}}\right)\right] \\
& -\frac{\lambda}{2}\left[\left(a_{j+\frac{1}{2}} \sigma_{j+\frac{1}{2}}+u_{j+\frac{1}{2}}^{-} \sigma_{j+\frac{1}{2}}^{-}\right) h_{j+\frac{1}{2}}^{-}+\left(a_{j-\frac{1}{2}} \sigma_{j-\frac{1}{2}}-u_{j-\frac{1}{2}}^{+} \sigma_{j-\frac{1}{2}}^{+}\right) h_{j-\frac{1}{2}}^{+}\right. \\
& \left.+a_{j+\frac{1}{2}} \sigma_{j+\frac{1}{2}} B\left(x_{j+\frac{1}{2}}\right)+a_{j-\frac{1}{2}} \sigma_{j-\frac{1}{2}} B\left(x_{j-\frac{1}{2}}\right)\right] .
\end{aligned}
$$

The terms involving $\sigma_{j \pm \frac{1}{2}} B\left(x_{j \pm \frac{1}{2}}\right)$ on the right hand side cancel, and since $a_{j \pm \frac{1}{2}} \sigma_{j \pm \frac{1}{2}} \geq\left|u_{j \pm \frac{1}{2}}^{ \pm}\right| \sigma_{j \pm \frac{1}{2}}^{ \pm}$, (2.30), and $h_{j \pm \frac{1}{2}}^{\mp} \geq 0$, the $C F L$ restriction (2.35) allows us to write

$$
\bar{A}_{j}^{T}(t+\Delta t) \geq \bar{A}_{j}^{T}(t)-\frac{1}{2} \sigma_{j}\left(h_{j+\frac{1}{2}}^{-}+h_{j-\frac{1}{2}}^{+}\right)=\frac{1}{2} \sigma_{j}\left(B\left(x_{j+\frac{1}{2}}\right)+B\left(x_{\left.j-\frac{1}{2}\right)}\right),\right.
$$

from where (2.36) follows. And since the Runge-Kutta solver (2.31)-(2.32) consists of a convex combination of forward Euler steps, the result holds when this evolution routine is employed.

Acknowledgements. Work supported in part by NSF, Award number DMS 0609766. The authors would like to thank Alfredo N. Wetzel (Department of Mathematics and Aerospace Engineering, University of Michigan) for helping us compute exact solutions. 


\section{REFERENCES}

[1] R. Abgrall and S. Karni, A relaxation scheme for the two layer shallow water system, in Proceedings of the 11th International Conference on Hyperbolic Problems (Lyon, 2006), Springer (2008) 135-144.

[2] E. Audusse, F. Bouchut, M.-O. Bristeau, R. Klein and B. Perthame, A fast and stable well-balanced scheme with hydrostatic reconstruction for shallow water flows. SIAM J. Sci. Comput. 25 (2004) 2050-2065

[3] J. Balbás and E. Tadmor, Nonoscillatory central schemes for one- and two-dimensional magnetohydrodynamics equations. ii: High-order semidiscrete schemes. SIAM J. Sci. Comput. 28 (2006) 533-560.

[4] A. Bermudez and M.E. Vazquez, Upwind methods for hyperbolic conservation laws with source terms. Comput. Fluids 23 (1994) 1049-1071.

[5] F. Bouchut, Nonlinear stability of finite volume methods for hyperbolic conservation laws and well-balanced schemes for sources. Birkhauser, Basel, Switzerland, Berlin (2004).

[6] M.J. Castro, J. Macias and C. Pares, A Q-scheme for a class of systems of coupled conservation laws with source terms. Application to a two-layer 1-d shallow water system. ESAIM: M2AN 35 (2001) 107-127.

[7] M.J. Castro, J.A. García-Rodríguez, J.M. González-Vida, J. Macías, C. Parés and M.E. Vázquez-Cendón, Numerical simulation of two-layer shallow water flows through channels with irregular geometry. J. Comput. Phys. 195 (2004) 202-235.

[8] N. Črnjarić-Žic, S. Vuković and L. Sopta, Balanced finite volume WENO and central WENO schemes for the shallow water and the open-channel flow equations. J. Comput. Phys. 200 (2004) 512-548.

[9] S. Gottlieb, C.-W. Shu and E. Tadmor, Strong stability-preserving high-order time discretization methods. SIAM Rev. 43 (2001) 89-112.

[10] J.M. Greenberg and A.Y. Le Roux, Well-balanced scheme for the processing of source terms in hyperbolic equations. SIAM J. Numer. Anal. 33 (1996) 1-16.

[11] A. Harten, High resolution schemes for hyperbolic conservation laws. J. Comput. Phys. 49 (1983) 357-393.

[12] S. Jin, A steady-state capturing method for hyperbolic systems with geometrical source terms. ESAIM: M2AN 35 (2001) 631-645.

[13] A. Kurganov and D. Levy, Central-upwind schemes for the Saint-Venant system. ESAIM: M2AN 36 (2002) $397-425$.

[14] A. Kurganov and G. Petrova, A second-order well-balanced positivity preserving central-upwind scheme for the Saint-Venant system. Commun. Math. Sci. 5 (2007) 133-160.

[15] A. Kurganov and E. Tadmor, New high-resolution central schemes for nonlinear conservation laws and convection-diffusion equations. J. Comput. Phys. 160 (2000) 241-282.

[16] A. Kurganov, S. Noelle and G. Petrova, Semidiscrete central-upwind schemes for hyperbolic conservation laws and HamiltonJacobi equations. SIAM J. Sci. Comput. 23 (2001) 707-740.

[17] R.J. LeVeque, Balancing source terms and flux gradients in high resolution Godunov methods: the quasi-steady wavepropagation algorithm. J. Comp. Phys. 146 (1998) 346-365.

[18] H. Nessyahu and E. Tadmor, Nonoscillatory central differencing for hyperbolic conservation laws. J. Comput. Phys. 87 (1990) 408-463.

[19] S. Noelle, N. Pankratz, G. Puppo and J.R. Natvig, Well-balanced finite volume schemes of arbitrary order of accuracy for shallow water flows. J. Comput. Phys. 213 (2006) 474-499.

[20] S. Noelle, Y. Xing, and C.-W. Shu, High-order well-balanced finite volume WENO schemes for shallow water equation with moving water. J. Comput. Phys. 226 (2007) 29-58.

[21] C. Pares and M. Castro, On the well-balance property of Roe's method for nonconservative hyperbolic systems. Applications to shallow-water systems. ESAIM: M2AN 38 (2004) 821-852.

[22] B. Perthame and C. Simeoni, A kinetic scheme for the Saint-Venant system with a source term. Calcolo 38 (2001) $201-231$.

[23] G. Russo, Central schemes for balance laws, in Hyperbolic problems: theory, numerics, applications, Vols. I, II (Magdeburg, 2000), Internat. Ser. Numer. Math. 140, Birkhäuser, Basel (2001) 821-829.

[24] C.-W. Shu and S. Osher, Efficient implementation of essentially nonoscillatory shock-capturing schemes. II. Comput. Phys. 83 (1989) 32-78.

[25] W.C. Thacker, Some exact solutions to the nonlinear shallow-water wave equations. Journal of Fluid Mechanics Digital Archive 107 (1981) 499-508.

[26] B. van Leer, Towards the ultimate conservative difference scheme. V. A second-order sequel to Godunov's method. J. Comput. Phys. 135 (1997) 229-248.

[27] M.E. Vázquez-Cendón, Improved treatment of source terms in upwind schemes for the shallow water equations in channels with irregular geometry. J. Comput. Phys. 148 (1999) 497-526.

[28] S. Vuković and L. Sopta, High-order ENO and WENO schemes with flux gradient and source term balancing, in Applied mathematics and scientific computing (Dubrovnik, 2001), Kluwer/Plenum, New York (2003) 333-346. 